\title{
OPTIMAL-ORDER ERROR ESTIMATES FOR THE FINITE ELEMENT APPROXIMATION OF THE SOLUTION OF A NONCONVEX VARIATIONAL PROBLEM
}

\author{
CHARLES COLLINS AND MITCHELL LUSKIN
}

\begin{abstract}
Nonconvex variational problems arise in models for the equilibria of crystals and other ordered materials. The solution of these variational problems must be described in terms of a microstructure rather than in terms of a deformation. Moreover, the numerical approximation of the deformation gradient often does not converge strongly as the mesh is refined. Nevertheless, the probability distribution of the deformation gradients near each material point does converge. Recently we introduced a metric to analyze this convergence. In this paper, we give an optimal-order error estimate for the convergence of the deformation gradient in a norm which is stronger than the metric used earlier.
\end{abstract}

\section{INTRODUCTION}

Nonconvex variational problems often arise in the modeling of the equilibria of crystals or other ordered states [2-9], [11-20]. For instance, the free energy for a solid crystal which has symmetry-related (martensitic) variants will have multiple, distinct energy wells. These variational problems may fail to attain a minimum value for any admissible deformation. Rather, the deformation gradients of minimizing sequences can have oscillations which do not converge strongly enough to evaluate nonlinear integrals of the deformation gradient such as the bulk energy functional. Nevertheless, the solution to these variational problems can be described in terms of an appropriate mathematical description of microstructure such as the Young measure [2-5], [15-20].

A continuum theory to describe the equilibria of crystals such as CuZn, $\mathrm{CuAlNi}, \mathrm{NiAl}$, and InTl which have symmetry-related variants has been recently developed [2-9], [11-20]. A corresponding theory of microstructure using the concept of the Young measure, or parametrized measure, has also been recently

\footnotetext{
Received June 21, 1990. $73 \mathrm{C} 60$.

1980 Mathematics Subject Classification(1985 Revision). Primary 65N15, 65N30, 35J20, 35J70,

Key words and phrases. Finite element method, error estimates, numerical approximation, variational problem, nonconvex.

This work is part of the Transitions and Defects in Ordered Materials Project and was supported in part by the National Science Foundation and the Air Force Office of Scientific Research through grants DMS 835-1080 and DMS 871-8881, the Army Research Office through grants DAAL03-88-K0110 and DAAL03-89-G-0081, the Cray Research Foundation, and by a grant from the Minnesota Supercomputer Institute.
} 
developed to describe solutions to the variational problems given by the above continuum theory [2-5], [15-20]. This theory of microstructure gives a calculus for the computation of macroscopic properties of the crystals.

We have reported computational results for two- and three-dimensional models which give oscillations in the gradient on the scale of the mesh [7-9]. These oscillations do not converge strongly in any $L^{p}$ space, even locally, as the mesh is refined. However, the probability distribution of the deformation gradients near each material point does converge to a Young measure (or parametrized measure). We introduced a metric to analyze this convergence in [6], and we obtained a $O\left(h^{1 / 4}\right)$ convergence rate in this metric for a one-dimensional model problem by obtaining error estimates for the probability distribution of the deformation gradient near each point. In this paper, we utilize some new analytic methods to obtain an optimal-order $O(h)$ error estimate for the deformation gradient in a norm with a stronger topology than the metric in [6] as well as other improved estimates.

We define the mathematical problem and the norm in $\S 2$, and we give the main results in that section. In $\S 3$ we prove the main results for problems with unconstrained boundary conditions. We give the extension of these results to the Dirichlet problem in $\S 4$. The optimality of the order of the error estimates is given in $\S 5$.

\section{CONVERGENCE OF THE DEFORMATION GRADIENT}

We denote by $L^{p}$ for $1 \leq p \leq \infty$ with norm $|v|_{L^{p}}$ the usual space of Lebesgue measurable functions [21] on $I \equiv(0,1)$ such that

$$
|v|_{L^{p}} \equiv\left[\int_{0}^{1}|v(x)|^{p} d x\right]^{1 / p}<\infty \quad \text { for } 1 \leq p<\infty
$$

and

$$
|v|_{L^{\infty}} \equiv \underset{x \in I}{\operatorname{ess} \sup }|v(x)|<\infty .
$$

We then denote the Sobolev space $H^{1}$ by $[1$, p. 44]

$$
H^{1}=\left\{v \in L^{2}: v^{\prime} \in L^{2}\right\} .
$$

The energy density $\phi(s)$ for our model of a one-dimensional crystal satisfies

$$
\begin{gathered}
\lambda_{1} \min \left\{\left(s-s_{L}\right)^{2},\left(s-s_{U}\right)^{2}\right\} \leq \phi(s) \leq \lambda_{2}(|s|+1)^{2} \quad \text { for all } s \in \mathbb{R}, \\
\phi\left(s_{L}\right)=\phi\left(s_{U}\right)=0,
\end{gathered}
$$

where $\lambda_{1}$ and $\lambda_{2}$ are material constants, $s$ is the linear strain, and $s_{L}$ and $s_{U}$ with $s_{L}<s_{U}$ represent the transformation strains for the martensitic variants. We note that by Lemma 2 it follows that the energy density need be defined (and satisfy) (2.1) only in a neighborhood of $\left\{s_{L}, s_{U}\right\}$. A derivation of the energy density (2.1) from a three-dimensional physical model with one-dimensional 
symmetry is given in [2]. We model the bulk energy of a martensitic onedimensional crystal [2] by

$$
\mathscr{E}(v)=\int_{0}^{1}\left[\phi\left(v^{\prime}(y)\right)+(v(y)-f(y))^{2}\right] d y,
$$

where $v(y)$ is a scalar-valued displacement and

$$
f(y)=\bar{s} y+b \quad \text { for } y \in I
$$

for $\bar{s}$ satisfying $s_{L}<\bar{s}<s_{U}$. The functional $\mathscr{E}(v)$ is well-defined for $v \in H^{1}$. It is well known (see Lemma 1 below, for instance) that

$$
\inf _{v \in H^{1}} \mathscr{E}(v)=0
$$

but that there does not exist $u \in H^{1}$ such that $\mathscr{E}(u)=0$. (To see this, assume that $\mathscr{E}(u)=0$. Since $\int_{0}^{1}(u(x)-f(x))^{2} d x=0$, we have that $u(x)=f(x)$ a.e. Thus, $\phi\left(u^{\prime}\right)=\phi\left(f^{\prime}\right)=\phi(\bar{s})>0$. So, $\int_{0}^{1} \phi\left(u^{\prime}(x)\right) d x>0$. This contradicts the assumption that $\mathscr{E}(u)=0$.)

We shall give an optimal-order error analysis for the minimization of the functional $\mathscr{E}$ over finite element spaces, $\mathscr{M}_{h}$. To define $\mathscr{M}_{h}$, let the mesh length $h=1 / M$ for some $M \in \mathbb{N}$; let the vertex points $x_{i}=i h$ for $i=0, \ldots, M$; and let the subintervals $I_{i}=\left(x_{i-1}, x_{i}\right)$ for $i=1, \ldots, M$. The finite element space $\mathscr{M}_{h}$ is defined to be the space of piecewise linear, continuous functions

$$
\mathscr{M}_{h} \equiv\left\{v \in C(I):\left.v\right|_{I_{i}} \text { is linear for } i=1, \ldots, M\right\} .
$$

The approximate solutions $u_{h} \in \mathscr{M}_{h}$ satisfy

$$
\mathscr{E}\left(u_{h}\right)=\min _{v_{h} \in \mathscr{M}_{h}} \mathscr{E}\left(v_{h}\right) \equiv E_{h} .
$$

The following lemma was proven in [6]. For completeness, we give a more elementary proof in $\S 3$.

Lemma 1. The energy $E_{h}$ converges to 0 at the rate given by

$$
E_{h} \leq \frac{\left(s_{U}-s_{L}\right)^{2} h^{2}}{4} .
$$

However, $u_{h}^{\prime}(x)$ does not converge as $h \rightarrow 0$ in any $L^{p}$ space, even locally. In [6], it was shown that $u_{h}^{\prime}(x)$ and nonlinear functions of $u_{h}^{\prime}(x)$ converge weakly, though. We introduced a metric for this convergence in [6], and we showed that the convergence rate was $O\left(h^{1 / 4}\right)$. In this paper, we give a proof that the convergence rate is $O(h)$ in a norm with a stronger topology than the metric in [6] and we show that this convergence rate is optimal.

Before we define the norm for the convergence of $u_{h}^{\prime}(x)$ we need to recall that we proved in [6] that $u_{h}^{\prime}(x)$ oscillates about a small neighborhood of $s_{L}$ and $s_{U}$. More precisely, we proved a variant of the following lemma in [6] which we also review in $\S 3$. 
Lemma 2. The approximate strain $u_{h}^{\prime}(x)$ satisfies the bound

$$
\max _{x \in I} \min \left\{\left(u_{h}^{\prime}(x)-s_{L}\right)^{2},\left(u_{h}^{\prime}(x)-s_{U}\right)^{2}\right\} \leq \frac{\left(s_{U}-s_{L}\right)^{2} h}{4 \lambda_{1}} .
$$

For $\hat{s} \in \mathbb{R}$ and $\alpha>0$ denote the closed ball of radius $\alpha$ at $\hat{s}$ by

$$
\mathscr{B}_{\alpha}(\hat{s})=\{s: \hat{s}-\alpha \leq s \leq \hat{s}+\alpha\}
$$

and denote the closed neighborhood $\mathscr{N}_{\alpha}$ of $\left\{s_{L}, s_{U}\right\}$ by

$$
\mathscr{N}_{\alpha}=\mathscr{B}_{\alpha}\left(s_{L}\right) \cup \mathscr{B}_{\alpha}\left(s_{U}\right) .
$$

It follows from Lemma 2 that if $h \leq 4 \alpha^{2} \lambda_{1} /\left(s_{U}-s_{L}\right)^{2}$, then

$$
u_{h}^{\prime}(x) \in \mathscr{N}_{\alpha} \quad \text { for } x \in I .
$$

Equivalently,

$$
u_{h}^{\prime}(x) \in \mathscr{N}_{h^{1 / 2}\left(s_{U}-s_{L}\right) / 2 \lambda_{1}^{1 / 2}} \quad \text { for } x \in I .
$$

Our first main theorem states that the approximate strains, $u_{h}^{\prime}(x)$, are locally in the state $s_{L}$ with probability

$$
\gamma=\frac{s_{U}-\bar{s}}{s_{U}-s_{L}}
$$

and are locally in the state $s_{U}$ with probability $1-\gamma=\left(\bar{s}-s_{L}\right) /\left(s_{U}-s_{L}\right)$. In the jargon of the calculus of variations, the measure

$$
\nu_{x} \equiv \gamma \delta_{s_{L}}+(1-\gamma) \delta_{s_{U}} \quad \text { for } 0<x<1,
$$

where $\delta_{\hat{s}}$ is the Dirac delta function with unit mass concentrated at $\hat{s}$ for $\hat{s}=s_{L}, s_{U}$, is the unique Young measure associated with minimizing (2.2).

We define the Sobolev space $\mathscr{V}$ to be the space of functions $F(x, s): I \times$ $\mathscr{N}_{\alpha} \rightarrow \mathbb{R}$ such that

$$
\frac{\partial F}{\partial s} \in L^{2}\left(I, L^{\infty}\left(\mathscr{N}_{\alpha}\right)\right)
$$

and

$$
\frac{\partial}{\partial x}\left[F\left(x, s_{U}\right)-F\left(x, s_{L}\right)\right] \in L^{1}(I)
$$

with norm (which depends on $\alpha>0$ )

$$
\begin{aligned}
\|F\|_{\mathscr{V}}= & {\left[\int_{0}^{1}\left|\frac{\partial F}{\partial s}(x, \cdot)\right|_{L^{\infty}\left(\mathcal{N}_{\alpha}\right)}^{2} d x\right]^{1 / 2}+\int_{0}^{1} \int_{\mathcal{N}_{\alpha}}|F(x, s)| d s d x } \\
& +\left|F\left(0, s_{U}\right)-F\left(0, s_{L}\right)\right|+\int_{0}^{1}\left|\frac{\partial}{\partial x}\left[F\left(x, s_{U}\right)-F\left(x, s_{L}\right)\right]\right| d x .
\end{aligned}
$$

We will prove the following theorem. 
Theorem 1. For $h \leq \min \left\{4 \lambda_{1}, 4 \alpha^{2} \lambda_{1} /\left(s_{U}-s_{L}\right)^{2}\right\}$ and $F \in \mathscr{V}$ we have the estimate

$$
\begin{aligned}
\left|\int_{0}^{1}\left[F\left(x, u_{h}^{\prime}(x)\right)-\gamma F\left(x, s_{L}\right)-(1-\gamma) F\left(x, s_{U}\right)\right] d x\right| \\
\leq\left[\frac{\left(s_{U}-s_{L}\right)}{2 \lambda_{1}^{1 / 2}}+\frac{1}{2 \lambda_{1}^{1 / 2}}+4\right]\|F\|_{\mathscr{V}} h .
\end{aligned}
$$

We note that the thermodynamic properties of materials depend nonlinearly on the strain $s$. Theorem 1 shows that even though $u_{h}(x) \rightarrow f(x)$ uniformly as $h \rightarrow 0$ (see Lemma 5 ), the material property described by the microscopic density $F(y, s)$ has the macroscopic density (weak limit)

$$
\gamma F\left(x, s_{L}\right)+(1-\gamma) F\left(x, s_{U}\right)
$$

for the minimizing microstructure for the energy (2.2).

To estimate the rate of convergence of $u_{h}^{\prime}(x)$ we define the operator norm on the dual $\mathscr{V}^{*}$ of $\mathscr{V}$ by

$$
\|L\|_{\mathscr{V}^{*}}=\sup _{F \in \mathscr{V}} \frac{|\langle L, F\rangle|}{\|F\|_{\mathscr{V}}}
$$

for $L \in \mathscr{V}^{*}$. For $h \leq 4 \alpha^{2} \lambda_{1} /\left(s_{U}-s_{L}\right)^{2}$, we can identify with $u_{h}^{\prime}$ the functional $L_{u_{h}^{\prime}} \in \mathscr{V}^{*}$ defined by

$$
\left\langle L_{u_{h}^{\prime}}, F\right\rangle \equiv \int_{0}^{1} F\left(x, u_{h}^{\prime}\right) d x,
$$

and we identify with $\nu \equiv \gamma \delta_{s_{L}}+(1-\gamma) \delta_{s_{U}}$ the functional $L_{\nu} \in \mathscr{V}^{*}$ defined by

$$
\left\langle L_{\nu}, F\right\rangle \equiv \int_{0}^{1}\left[\gamma F\left(x, s_{L}\right)+(1-\gamma) F\left(x, s_{U}\right)\right] d x .
$$

We then have the following theorem which is a direct consequence of (2.8).

Theorem 2. For $h \leq \min \left\{4 \lambda_{1}, 4 \alpha^{2} \lambda_{1} /\left(s_{U}-s_{L}\right)^{2}\right\}$ we have that

$$
\left\|L_{u_{h}^{\prime}}-L_{\nu}\right\|_{\mathscr{V}^{*}} \leq\left[\frac{\left(s_{U}-s_{L}\right)}{2 \lambda_{1}^{1 / 2}}+\frac{1}{2 \lambda_{1}^{1 / 2}}+4\right] h .
$$

We also have that

$$
\left\langle L_{u_{h}^{\prime}}, F\right\rangle \leq\|F\|_{L^{1}\left(I, C\left(\mathcal{N}_{\alpha}\right)\right)} \quad \text { for } F \in L^{1}\left(I, C\left(\mathscr{N}_{\alpha}\right)\right)
$$

Thus, $L_{u_{h}^{\prime}}$ is uniformly bounded in the operator norm (with norm 1 ) in the dual of $L^{1}\left(I, C\left(\mathscr{N}_{\alpha}\right)\right)$. Since $\mathscr{V}$ is dense in $L^{1}\left(I, C\left(\mathscr{N}_{\alpha}\right)\right)$, the above result implies that $L_{u_{h}^{\prime}} \rightarrow L_{\nu}$ as $h \rightarrow 0$ in the weak*-topology of $L^{1}\left(I, C\left(\mathscr{N}_{\alpha}\right)\right)^{*}$. It is known $[10,22]$ that

$$
L^{1}\left(I, C\left(\mathscr{N}_{\alpha}\right)\right)^{*}=L^{\infty}\left(I, C\left(\mathscr{N}_{\alpha}\right)^{*}\right)=L^{\infty}\left(I, M\left(\mathscr{N}_{\alpha}\right)\right)
$$


where $M\left(\mathscr{N}_{\alpha}\right)$ is the space of real Borel measures on $\mathscr{N}_{\alpha}$ with bounded variation.

Results similar to those described above are given in $\S 4$ for the Dirichlet problem to minimize $\mathscr{E}(v)$ over $\mathscr{W}$ where

$$
\mathscr{W} \equiv\left\{v \in H^{1}: v(0)=f(0) \text { and } v(1)=f(1)\right\} .
$$

We note that the variational problem

$$
\inf _{v \in \mathscr{W}} \int_{0}^{1} \phi\left(v^{\prime}\right) d y
$$

may have many solutions, both in the sense that the limit of the displacements $v(x)$ of a minimizing sequence need not be unique and in the sense that the possible Young measure for the strains $v^{\prime}(x)$ need not be unique. However, the simplest limit displacement in this case is affine and the unconstrained problem

$$
\inf _{v \in H^{1}} \mathscr{E}(v)
$$

has this as its unique limit displacement. Moreover, in our present situation, the Young measure so generated is also unique. Since the multidimensional Dirichlet problem corresponding to (2.11) gives a unique Young measure for appropriate affine boundary conditions [4], we utilize the term

$$
\int_{0}^{1}(v(y)-f(y))^{2} d y
$$

in the definition of $\mathscr{E}(v)$ to select a unique Young measure analogous to the selection of a unique Young measure for multidimensional problems by appropriate Dirichlet boundary conditions. Thus, we consider (2.2) in place of the more traditional variational integral. A mechanical interpretation of the term (2.13) can be obtained from a model of a thin crystal plate glued to a rigid substrate [2].

\section{ERROR ESTIMATES FOR THE DEFORMATION GRADIENT}

We can assume in the following without loss of generality that

$$
f(y) \equiv 0
$$

and

$$
s_{L}<0<s_{U}
$$

To see this, note that $v_{h}(x)+f(x) \in \mathscr{M}_{h}$ if $v_{h} \in \mathscr{M}_{h}$ and that

$$
\mathscr{E}(v+f)=\int_{0}^{1}\left[\phi\left(v^{\prime}(y)+\bar{s}\right)+v(y)^{2}\right] d y .
$$

Further, $\phi(s+\bar{s})$ satisfies $(2.1)$ with $s_{L}$ replaced by $s_{L}+\bar{s}$ and $s_{U}$ replaced by $s_{U}+\bar{s}$ if $\phi(s)$ satisfies (2.1). We first give a simplified proof of Lemma 1 [6]. 
Proof of Lemma 1. Define $v_{h}(x) \in \mathscr{M}_{h}$ by $v_{h}(0)=0$ and for $k=0, \ldots$, $M-1$,

$$
v_{h}\left(x_{k+1}\right)= \begin{cases}v_{h}\left(x_{k}\right)+h s_{L} & \text { if }\left|v_{h}\left(x_{k}\right)+h s_{L}\right| \leq\left|v_{h}\left(x_{k}\right)+h s_{U}\right|, \\ v_{h}\left(x_{k}\right)+h s_{U} & \text { if }\left|v_{h}\left(x_{k}\right)+h s_{L}\right|>\left|v_{h}\left(x_{k}\right)+h s_{U}\right| .\end{cases}
$$

Since $v_{h}^{\prime}(x)=s_{L}$ or $v_{h}^{\prime}(x)=s_{U}$ for $x \in I$ we have that $\phi\left(v_{h}^{\prime}(x)\right) \equiv 0$.

Next, we shall show that

$$
v_{h}(x) \leq\left(s_{U}-s_{L}\right) h / 2 \quad \text { for } x \in I .
$$

Suppose there were a smallest positive integer $P$ such that

$$
v_{h}\left(x_{P}\right)>\left(s_{U}-s_{L}\right) h / 2 \text {. }
$$

Since $v_{h}\left(x_{P}\right)>v_{h}\left(x_{P-1}\right)$, it follows from the definition of $v_{h}(x)$ above that

$$
v_{h}\left(x_{P}\right)=v_{h}\left(x_{P-1}\right)+h s_{U} .
$$

However, by (3.1),

$$
v_{h}\left(x_{P}\right)=v_{h}\left(x_{P-1}\right)+h s_{U}>\left(s_{U}-s_{L}\right) h / 2,
$$

or equivalently, after some elementary algebra,

$$
\left|v_{h}\left(x_{P-1}\right)+h s_{U}\right|=v_{h}\left(x_{P-1}\right)+h s_{U}>-v_{h}\left(x_{P-1}\right)-h s_{L} .
$$

Since $s_{L}<s_{U}$ we also have that

$$
\left|v_{h}\left(x_{P-1}\right)+h s_{U}\right|=v_{h}\left(x_{P-1}\right)+h s_{U}>v_{h}\left(x_{P-1}\right)+h s_{L} .
$$

Thus, the previous two inequalities imply that

$$
\left|v_{h}\left(x_{P-1}\right)+h s_{U}\right|>\left|v_{h}\left(x_{P-1}\right)+h s_{L}\right| \text {. }
$$

This contradicts the definition of $v_{h}(x)$. The proof that

$$
-v_{h}(x) \leq\left(s_{U}-s_{L}\right) h / 2 \quad \text { for } x \in I
$$

is similar. Thus, we have shown that

$$
\left|v_{h}(x)\right| \leq\left(s_{U}-s_{L}\right) h / 2 \quad \text { for } x \in I .
$$

Hence, it follows that

$$
E_{h} \leq \mathscr{E}\left(v_{h}\right) \leq \max \left|v_{h}\right|^{2} \leq \frac{\left(s_{U}-s_{L}\right)^{2} h^{2}}{4} .
$$

We next prove Lemma 2 which gives a pointwise estimate of the oscillation of $u_{h}^{\prime}(x)$ about $s_{L}$ and $s_{U}$.

Proof of Lemma 2. Since $u_{h}^{\prime}(x)$ is piecewise constant on the finite element mesh of length scale $h$, it follows from (2.1) and Lemma 1 that

$$
\lambda_{1} \min \left\{\left(u_{h}^{\prime}(x)-s_{L}\right)^{2},\left(u_{h}^{\prime}(x)-s_{U}\right)^{2}\right\} h \leq \phi\left(u_{h}^{\prime}(x)\right) h \leq \frac{\left(s_{U}-s_{L}\right)^{2} h^{2}}{4}
$$

for $x \in I$. The result (2.4) follows directly from the above estimate. 
The next lemma gives an $L^{2}$ estimate of the oscillation of $u_{h}^{\prime}(x)$ about $s_{L}$ and $s_{U}$. It will be useful to have the projection operator $\Pi: \mathbb{R} \rightarrow\left\{s_{L}, s_{U}\right\}$ defined by

Note that

$$
\Pi s= \begin{cases}s_{L} & \text { for } s<\left(s_{L}+s_{U}\right) / 2 \\ s_{U} & \text { for } s \geq\left(s_{L}+s_{U}\right) / 2\end{cases}
$$

$$
|s-\Pi s|^{2}=\min \left\{\left(s-s_{L}\right)^{2},\left(s-s_{U}\right)^{2}\right\},
$$

so by $(2.1)$

$$
\phi(s) \geq \lambda_{1}|s-\Pi s|^{2} .
$$

Lemma 3. We have the estimate

$$
\int_{0}^{1}\left|u_{h}^{\prime}(x)-\Pi u_{h}^{\prime}(x)\right|^{2} d x \leq \frac{\left(s_{U}-s_{L}\right)^{2} h^{2}}{4 \lambda_{1}} .
$$

Proof. The estimate follows easily from (2.1) and Lemma 1, since they imply that

$$
\lambda_{1} \int_{0}^{1}\left|u_{h}^{\prime}(x)-\Pi u_{h}^{\prime}(x)\right|^{2} d x \leq E_{h} \leq \frac{\left(s_{U}-s_{L}\right)^{2} h^{2}}{4} .
$$

Lemma 4. We have the bound

Proof. Set

$$
\max _{x \in I}\left|u_{h}(x)\right| \leq \max \left\{\max _{x \in I}\left|u_{h}^{\prime}(x)\right|, s_{U},\left|s_{L}\right|\right\} h .
$$

$$
\nu=\max \left\{\max _{x \in I}\left|u_{h}^{\prime}(x)\right|, s_{U},\left|s_{L}\right|\right\} .
$$

We assume that

$$
\max _{x \in I}\left|u_{h}(x)\right|>\nu h
$$

and we shall show that this leads to a contradiction by constructing $\hat{u}_{h}(x) \in \mathscr{M}_{h}$ such that

$$
\mathscr{E}\left(\hat{u}_{h}\right)<\mathscr{E}\left(u_{h}\right) .
$$

By (3.5), there exists $p$ such that $\left|u_{h}\left(x_{p}\right)\right| \geq\left|u_{h}\left(x_{l}\right)\right|$ for $l=0, \ldots, M$ and $\left|u_{h}\left(x_{p}\right)\right|>\nu h$. First, we show that $x_{p} \neq 0$ and $x_{p} \neq 1$. If $u_{h}(0)>\nu h$, then we construct $\hat{u}_{h}(x) \in \mathscr{M}_{h}$ by

$$
\hat{u}_{h}\left(x_{k}\right)= \begin{cases}u_{h}\left(x_{1}\right)-s_{U} h & \text { for } k=0, \\ u_{h}\left(x_{k}\right) & \text { for } k=1, \ldots, M .\end{cases}
$$

Now $u_{h}\left(x_{1}\right)>0$ since $u_{h}(0)>\nu h$ and since $\left|u_{h}^{\prime}(x)\right| \leq \nu$ for $x \in I_{1}$. Also, $\hat{u}_{h}(0)=u_{h}\left(x_{1}\right)-s_{U} h>-\nu h$, so $\left|\hat{u}_{h}(x)\right|<u_{h}(x)$ for $x \in I_{1}$. Further, $\hat{u}_{h}^{\prime}(x)=$ $s_{U}$ for $x \in I_{1}$ and $\hat{u}_{h}(x)=u_{h}(x)$ for $x \in\left(x_{1}, 1\right)$. Thus, we have that

$$
\mathscr{E}\left(\hat{u}_{h}\right)<\mathscr{E}\left(u_{h}\right) \text {. }
$$

Similar arguments for the other cases show that $x_{p} \neq 0$ and $x_{p} \neq 1$. 
We may now assume that $p$ is chosen so that $0<x_{p}<1$, that $\left|u_{h}\left(x_{p}\right)\right|>\nu h$, and that $\left|u_{h}\left(x_{p}\right)\right|>\left|u_{h}\left(x_{p-1}\right)\right|$ and $\left|u_{h}\left(x_{p}\right)\right| \geq\left|u_{h}\left(x_{p+1}\right)\right|$. In this case, we construct $\hat{u}_{h}(x) \in \mathscr{M}_{h}$ by

$$
\hat{u}_{h}\left(x_{k}\right)=\left\{\begin{array}{l}
u_{h}\left(x_{p-1}\right)+u_{h}\left(x_{p+1}\right)-u_{h}\left(x_{p}\right) \text { for } k=p, \\
u_{h}\left(x_{k}\right) \text { for } k=0, \ldots, p-1, p+1, \ldots, M .
\end{array}\right.
$$

Then $\hat{u}_{h}^{\prime}(x)=u_{h}^{\prime}(x+h)$ for $x \in I_{p}, \hat{u}_{h}^{\prime}(x)=u_{h}^{\prime}(x-h)$ for $x \in I_{p+1},\left|\hat{u}_{h}(x)\right|<$ $\left|u_{h}(x)\right|$ for $x \in I_{p} \cup I_{p+1}$, and $\hat{u}_{h}(x)=u_{h}(x)$ for $x \in\left(0, x_{p-1}\right) \cup\left(x_{p+1}, 1\right)$. Hence, we have that

$$
\mathscr{E}\left(\hat{u}_{h}\right)<\mathscr{E}\left(u_{h}\right)
$$

Thus, we have proved the lemma by contradiction.

Next, we give an estimate for the convergence of $u_{h}(x)$ to 0 . It is shown in $\S 5$ that this rate of convergence is optimal.

Lemma 5. If $h \leq 4 \lambda_{1}$, then

$$
\max _{x \in I}\left|u_{h}(x)\right| \leq 2\left(s_{U}-s_{L}\right) h
$$

Proof. It follows from Lemma 2 that if $h \leq 4 \lambda_{1}$, then

$$
\max _{x \in I}\left|u_{h}^{\prime}(x)\right| \leq 2\left(s_{U}-s_{L}\right) \text {. }
$$

The result (3.6) now follows from Lemma 4.

Proof of Theorem 1. We estimate the error as follows:

$$
\begin{aligned}
& \left|\int_{0}^{1}\left[F\left(x, u_{h}^{\prime}(x)\right)-\gamma F\left(x, s_{L}\right)-(1-\gamma) F\left(x, s_{U}\right)\right] d x\right| \\
& \leq\left|\int_{0}^{1}\left[F\left(x, u_{h}^{\prime}(x)\right)-F\left(x, \Pi u_{h}^{\prime}(x)\right)\right] d x\right| \\
& \quad+\left|\int_{0}^{1}\left[F\left(x, \Pi u_{h}^{\prime}(x)\right)-\gamma F\left(x, s_{L}\right)-(1-\gamma) F\left(x, s_{U}\right)\right] d x\right| \\
& =\mathscr{I}_{1}+\mathscr{I}_{2} .
\end{aligned}
$$

Then we have by Lemma 3 and the Cauchy-Schwarz inequality that

$$
\begin{aligned}
\mathscr{I}_{1} & \leq \int_{0}^{1}\left|\frac{\partial F}{\partial s}(x, \cdot)\right|_{L^{\infty}\left(\mathcal{N}_{\alpha}\right)}\left|u_{h}^{\prime}(x)-\Pi u_{h}^{\prime}(x)\right| d x \\
& \leq\left[\int_{0}^{1}\left|\frac{\partial F}{\partial s}(x, \cdot)\right|_{L^{\infty}\left(\mathcal{N}_{\alpha}\right)}^{2} d x\right]^{1 / 2}\left[\int_{0}^{1}\left|u_{h}^{\prime}(x)-\Pi u_{h}^{\prime}(x)\right|^{2} d x\right]^{1 / 2} \\
& \leq \frac{\left(s_{U}-s_{L}\right) h}{2 \lambda_{1}^{1 / 2}}\|F\|_{\mathscr{V}} .
\end{aligned}
$$


Let $G(x)=F\left(x, s_{L}\right)-F\left(x, s_{U}\right)$. If $\Pi u_{h}^{\prime}(x)=s_{L}$, then

$$
F\left(x, \Pi u_{h}^{\prime}(x)\right)-\gamma F\left(x, s_{L}\right)-(1-\gamma) F\left(x, s_{U}\right)=(1-\gamma) G(x)
$$

and if $\Pi u_{h}^{\prime}(x)=s_{U}$, then

$$
F\left(x, \Pi u_{h}^{\prime}(x)\right)-\gamma F\left(x, s_{L}\right)-(1-\gamma) F\left(x, s_{U}\right)=-\gamma G(x) .
$$

Note that by $(2.5)($ where $\bar{s}=0)$,

$$
\frac{\Pi u_{h}^{\prime}(x)}{s_{U}-s_{L}}= \begin{cases}\gamma-1 & \text { if } \Pi u_{h}^{\prime}(x)=s_{L}, \\ \gamma & \text { if } \Pi u_{h}^{\prime}(x)=s_{U}\end{cases}
$$

Thus, it follows from (3.7) and (3.8) that

$$
\begin{array}{r}
F\left(x, \Pi u_{h}^{\prime}(x)\right)-\gamma F\left(x, s_{L}\right)-(1-\gamma) F\left(x, s_{U}\right)=-\frac{\Pi u_{h}^{\prime}(x)}{s_{U}-s_{L}} G(x) \\
\text { for } x \in I .
\end{array}
$$

Hence, we can estimate $\mathscr{I}_{2}$ by

$$
\begin{aligned}
\mathscr{F}_{2} & =\left|\int_{0}^{1} \frac{\Pi u_{h}^{\prime}(x)}{s_{U}-s_{L}} G(x) d x\right| \\
& \leq \frac{1}{s_{U}-s_{L}}\left|\int_{0}^{1}\left(\Pi u_{h}^{\prime}(x)-u_{h}^{\prime}(x)\right) G(x) d x\right|+\frac{1}{s_{U}-s_{L}}\left|\int_{0}^{1} u_{h}^{\prime}(x) G(x) d x\right| \\
& =\frac{1}{s_{U}-s_{L}}\left(\mathscr{J}_{3}+\mathscr{I}_{4}\right) .
\end{aligned}
$$

Now by Lemma 3 and the Cauchy-Schwarz inequality we have that

$$
\begin{aligned}
\mathscr{F}_{3} & =\left|\int_{0}^{1}\left[u_{h}^{\prime}(x)-\Pi u_{h}^{\prime}(x)\right] G(x) d x\right| \\
& \leq\left\{\int_{0}^{1}\left[u_{h}^{\prime}(x)-\Pi u_{h}^{\prime}(x)\right]^{2} d x\right\}^{1 / 2}\left\{\int_{0}^{1} G^{2}(x) d x\right\}^{1 / 2} \\
& \leq \frac{\left(s_{U}-s_{L}\right) h}{2 \lambda_{1}^{1 / 2}}\|F\|_{\mathscr{V}},
\end{aligned}
$$

since

$$
\left\{\int_{0}^{1} G^{2}(x) d x\right\}^{1 / 2} \leq\|G\|_{L^{\infty}(I)} \leq|G(0)|+\int_{0}^{1}\left|\frac{\partial}{\partial x} G(x)\right| d x \leq\|F\|_{\mathscr{V}} .
$$


Next, use integration by parts on $\mathscr{F}_{4}$ and Lemma 5 to obtain that

$$
\begin{aligned}
\mathscr{F}_{4} & =\left|u_{h}(1) G(1)-u_{h}(0) G(0)-\int_{0}^{1} u_{h}(x) G^{\prime}(x) d x\right| \\
& \leq \max _{x \in I}\left|u_{h}(x)\right|\left[|G(1)|+|G(0)|+\int_{0}^{1}\left|G^{\prime}(x)\right| d x\right] \\
& \leq \max _{x \in I}\left|u_{h}(x)\right|\left[2|G(0)|+2 \int_{0}^{1}\left|G^{\prime}(x)\right| d x\right] \\
& \leq 4\left(s_{U}-s_{L}\right) h\|F\|_{\mathscr{V}} .
\end{aligned}
$$

Combining these results, we obtain that

$$
\begin{gathered}
\left|\int_{0}^{1}\left[F\left(x, u_{h}^{\prime}(x)\right)-\gamma F\left(x, s_{L}\right)-(1-\gamma) F\left(x, s_{U}\right)\right] d x\right| \\
\leq\left[\frac{\left(s_{U}-s_{L}\right)}{2 \lambda_{1}^{1 / 2}}+\frac{1}{2 \lambda_{1}^{1 / 2}}+4\right]\|F\|_{\mathscr{V}} h .
\end{gathered}
$$

\section{The Dirichlet PROBleM}

In this section we consider the numerical approximation of the Dirichlet problem to compute $u_{h} \in \mathscr{M}_{h} \cap \mathscr{W}$ satisfying

$$
\mathscr{E}\left(u_{h}\right)=\min _{v_{h} \in \mathscr{M}_{h} \cap \mathscr{W}} \mathscr{E}\left(v_{h}\right) .
$$

We further assume that there exist $\lambda_{2}$ and $\bar{\alpha}>0$ such that

$$
\phi(s) \leq \lambda_{2}|s-\Pi s|^{2} \quad \text { for } s \in \mathscr{N}_{\bar{\alpha}} .
$$

We can now prove the following variant of Lemma 1 for the Dirichlet problem.

Lemma 6. For $h \leq 2 \bar{\alpha} /\left(s_{U}-s_{L}\right)$, we have that

$$
\min _{v_{h} \in \mathscr{M}_{h} \cap \mathscr{\mathscr { V }}} \mathscr{E}\left(v_{h}\right) \leq\left(\frac{\lambda_{2}}{4}+\frac{2}{3}\right)\left(s_{U}-s_{L}\right)^{2} h^{2} .
$$

Proof. We define $w_{h} \in \mathscr{M}_{h} \cap \mathscr{W}$ by

$$
w_{h}(x)=v_{h}(x)-v_{h}(1) x
$$

where $v_{h}(x) \in \mathscr{M}_{h}$ is the function defined in the proof of Lemma 1. Now by (3.2),

$$
\begin{aligned}
\int_{0}^{1} w_{h}(x)^{2} d x & \leq 2 \int_{0}^{1}\left[v_{h}(x)^{2}+\left(v_{h}(x)-w_{h}(x)\right)^{2}\right] d x \\
& \leq \frac{2}{3}\left(s_{U}-s_{L}\right)^{2} h^{2} .
\end{aligned}
$$

Further, since $w_{h}^{\prime}(x) \in \mathscr{N}_{\bar{\alpha}}$ for $x \in I$, we have that

$$
\phi\left(w_{h}^{\prime}(x)\right) \leq \lambda_{2} v_{h}(1)^{2} \leq \frac{\lambda_{2}}{4}\left(s_{U}-s_{L}\right)^{2} h^{2}, \quad x \in I .
$$


Thus,

$$
\min _{v_{h} \in \mathscr{M}_{h} \cap \mathscr{W}} \mathscr{E}\left(v_{h}\right) \leq\left(\frac{\lambda_{2}}{4}+\frac{2}{3}\right)\left(s_{U}-s_{L}\right)^{2} h^{2} .
$$

Lemmas 2-5 and Theorems 1-2 for the Dirichlet problem can now be proven by the identical arguments used for problem (2.3). (The constants in these results must be changed to reflect the different constants in the bounds for the energy in Lemma 1 and Lemma 6.)

Finally, we note that the results of Lemmas 2-5 and Theorems 1-2 can be extended to the Dirichlet problem when the minimal energies attained in the wells of $\phi(s)$ are not equal. More specifically, we can allow the energy density $\phi(s)$ to have the property that $\hat{\phi}(s)=\phi(s)+l(s)$ satisfies $(2.1)$ where $l(s)$ is an affine function. To see this, note that if

$$
\hat{\mathscr{E}}(v)=\int_{0}^{1}\left[\hat{\phi}\left(v^{\prime}(y)\right)+(v(y)-f(y))^{2}\right] d y,
$$

then for $v \in \mathscr{W}$,

$$
\hat{\mathscr{E}}(v)=\mathscr{E}(v)+l(v(1)-v(0))=\mathscr{E}(v)+l(f(1)-f(0))
$$

Thus, $u_{h} \in \mathscr{M}_{h} \cap \mathscr{W}$ satisfies

$$
\mathscr{E}\left(u_{h}\right)=\min _{v_{h} \in \mathscr{M}_{h} \cap \mathscr{W}} \mathscr{E}\left(v_{h}\right)
$$

if and only if $u_{h} \in \mathscr{M}_{h} \cap \mathscr{W}$ satisfies

$$
\hat{\mathscr{E}}\left(u_{h}\right)=\min _{v_{h} \in \mathscr{M}_{h} \cap \mathscr{W}} \hat{\mathscr{E}}\left(v_{h}\right) .
$$

The analyses of Lemmas 1-5 and Theorems 1-2 can now be applied directly to problem (4.3).

\section{Optimality OF the MAIN RESUltS}

We next discuss the optimality of our results for problem (2.3). First, we set

$$
\tilde{\alpha}=\min \left\{-\frac{s_{L}}{2}, \frac{s_{U}}{2}\right\}>0
$$

It then follows from Lemma 2 that for $h \leq 4 \tilde{\alpha}^{2} \lambda_{1} /\left(s_{U}-s_{L}\right)^{2}$ we have that

$$
\left|u_{h}^{\prime}(x)\right| \geq \tilde{\alpha} \quad \text { for } x \in I .
$$

Hence, since $u_{h}^{\prime}(x)$ is linear on each interval $I_{i}$, we have for $i=1, \ldots, M$ that

$$
\max _{x \in I_{i}}\left|u_{h}(x)\right| \geq \tilde{\alpha} h / 2 \quad \text { for } x \in I_{i}
$$

and

$$
\int_{I_{i}} u_{h}(x)^{2} d x \geq \tilde{\alpha}^{2} h^{3} / 12
$$


Thus, from (5.2) we see that

$$
\int_{0}^{1} u_{h}(x)^{2} d x \geq \tilde{\alpha}^{2} h^{2} / 12
$$

and hence, that

$$
E_{h} \geq \tilde{\alpha}^{2} h^{2} / 12
$$

The estimate (5.4) shows the optimality of the order of the error bound for $E_{h}$ given in Lemma 1 and the estimate (5.1) shows the optimality of the order of the error bound for $u_{h}(x)$ given in Lemma 5 . Bounds similar to the above bounds are also clearly valid for the Dirichlet problem (4.1) .

To demonstrate the optimality of the bounds given in Theorem 1 and Theorem 2, we consider the example

$$
\phi(s)=(s-1)^{2}(s+1)^{2}
$$

for problem (2.3) In this case, $s_{L}=-1, s_{U}=1$, and $\gamma=1 / 2$. For this example, we can calculate analytically the displacement, $u_{h}(x)$, at which the minimum of the energy $\mathscr{E}$ is attained to be

$$
u_{h}\left(x_{i}\right)=(-1)^{i} \frac{h}{2}\left(1-\frac{h^{2}}{24}\right)^{1 / 2} \quad \text { for } i=0, \ldots, M .
$$

(The minimum energy is also attained at $-u_{h}(x)$, of course.) We also have that

$$
u_{h}^{\prime}(x)=(-1)^{i}\left(1-\frac{h^{2}}{24}\right)^{1 / 2} \quad \text { for } x \in I_{i}
$$

and

$$
\mathscr{E}\left(u_{h}\right)=\frac{h^{2}}{12}\left(1-\frac{h^{2}}{48}\right)
$$

To see this, note that the minimum of

$$
\mathscr{E}_{i}\left(v_{h}\right)=\int_{I_{i}}\left[\phi\left(v_{h}^{\prime}(y)\right)+v_{h}(y)^{2}\right] d y \quad \text { for } v_{h} \in \mathscr{M}_{h}
$$

is attained at $\tilde{u}_{h} \in \mathscr{M}_{h}$ such that

$$
\tilde{u}_{h}\left(x_{i-1}\right)= \pm \frac{h}{2}\left(1-\frac{h^{2}}{24}\right)^{1 / 2}, \quad \tilde{u}_{h}\left(x_{i}\right)=\mp \frac{h}{2}\left(1-\frac{h^{2}}{24}\right)^{1 / 2}
$$

with minimum energy

$$
\mathscr{E}_{i}\left(\tilde{u}_{h}\right)=\frac{h^{3}}{12}\left(1-\frac{h^{2}}{48}\right) .
$$

Hence, we see that the order of the bounds in Lemma 2 and Lemma 3 are not optimal for this example and that

$$
\int_{0}^{1}\left|u_{h}^{\prime}(x)-\Pi u_{h}^{\prime}(x)\right| d x \leq \frac{h^{2}}{24} .
$$


However, by asymptotic methods (nonrigorous) we have found that the order of the estimate in Lemma 3 is optimal for the Dirichlet problem. We have further found for the Dirichlet problem that in general the pointwise result $u_{h}^{\prime}(x)-\Pi u_{h}^{\prime}(x)=O(h)$ holds.

A review of the proof of Theorem 1 shows for the energy density (5.5) and problem (2.3) that

$$
\mathscr{I}_{1} \leq \beta_{1} h^{2} / 24 \quad \text { and } \quad \mathscr{I}_{3} \leq \beta_{2} h^{2} / 24 .
$$

Thus,

$$
\begin{aligned}
& \left|\int_{0}^{1}\left[F\left(x, u_{h}^{\prime}(x)\right)-\gamma F\left(x, s_{L}\right)-(1-\gamma) F\left(x, s_{U}\right)\right] d x\right| \\
& \quad \geq-\left[\frac{\beta_{1}}{24}+\frac{\beta_{2}}{24\left(s_{U}-s_{L}\right)}\right] h^{2} \\
& \quad+\frac{1}{s_{U}-s_{L}}\left|u_{h}(1) G(1)-u_{h}(0) G(0)-\int_{0}^{1} u_{h}(x) G^{\prime}(x) d x\right| .
\end{aligned}
$$

Now

$$
\int_{0}^{1} u_{h}(x) G^{\prime}(x) d x=\int_{0}^{1} u_{h}(x)\left(G^{\prime}(x)-\widehat{G}^{\prime}(x)\right) d x,
$$

where $\hat{G}^{\prime}(x)$ is the piecewise constant function which takes the average value of $G^{\prime}(x)$ on each interval $I_{i}$, defined by

$$
\widehat{G}^{\prime}(x)=\frac{1}{h} \int_{I_{i}} G^{\prime}(y) d y \quad \text { for } x \in I_{i},
$$

since

$$
\int_{I_{i}} u_{h}(x) d x=0 \quad \text { for } i=1, \ldots, M
$$

Further,

$$
\begin{aligned}
\mid \int_{0}^{1} u_{h}(x) & \left(G^{\prime}(x)-\widehat{G}^{\prime}(x)\right) d x \mid \\
& \leq \max _{x \in I}\left|u_{h}(x)\right| \int_{0}^{1}\left|G^{\prime}(x)-\widehat{G}^{\prime}(x)\right| d x \\
& \leq \max _{x \in I}\left|u_{h}(x)\right| h \int_{0}^{1}\left|G^{\prime \prime}(x)\right| d x \\
& \leq \frac{h^{2}}{2}\left(1-\frac{h^{2}}{24}\right)^{1 / 2} \int_{0}^{1}\left|G^{\prime \prime}(x)\right| d x
\end{aligned}
$$


Hence, by using the estimates (5.10) and (5.12) we obtain

$$
\begin{aligned}
& \left|\int_{0}^{1}\left[F\left(x, u_{h}^{\prime}(x)\right)-\gamma F\left(x, s_{L}\right)-(1-\gamma) F\left(x, s_{U}\right)\right] d x\right| \\
& \quad \geq-\left[\frac{\beta_{1}}{24}+\frac{\beta_{2}}{24\left(s_{U}-s_{L}\right)}+\frac{1}{2\left(s_{U}-s_{L}\right)}\left(1-\frac{h^{2}}{24}\right)^{1 / 2} \int_{0}^{1}\left|G^{\prime \prime}(x)\right| d x\right] h^{2} \\
& \quad+\frac{1}{s_{U}-s_{L}}\left|u_{h}(1) G(1)-u_{h}(0) G(0)\right| .
\end{aligned}
$$

Thus, for $G(0)=1$ and $G(1)=(-1)^{M+1}$, we have

$$
\left|u_{h}(1) G(1)-u_{h}(0) G(0)\right|=2\left|u_{h}(0)\right|=h\left(1-\frac{h^{2}}{24}\right)^{1 / 2}
$$

So, we finally obtain the estimate

$$
\begin{aligned}
& \left|\int_{0}^{1}\left[F\left(x, u_{h}^{\prime}(x)\right)-\gamma F\left(x, s_{L}\right)-(1-\gamma) F\left(x, s_{U}\right)\right] d x\right| \\
& \quad \geq-\left[\frac{\beta_{1}}{24}+\frac{\beta_{2}}{24\left(s_{U}-s_{L}\right)}+\frac{1}{2\left(s_{U}-s_{L}\right)}\left(1-\frac{h^{2}}{24}\right)^{1 / 2} \int_{0}^{1}\left|G^{\prime \prime}(x)\right| d x\right] h^{2} \\
& \quad+\frac{h}{s_{U}-s_{L}}\left(1-\frac{h^{2}}{24}\right)^{1 / 2}
\end{aligned}
$$

The above estimate shows that the order of the error bound in Theorem 1 (and, therefore, Theorem 2) is optimal.

We note that if $F(x, s)$ is independent of $x$, i.e., $F(x, s)=F(s)$, then $G(1)=G(0)$. Now if $M$ is even, we have that $u_{h}(0)=u_{h}(1)$, so

$$
u_{h}(1) G(1)-u_{h}(0) G(0)=0
$$

and

$$
\begin{aligned}
& \left|\int_{0}^{1}\left[F\left(x, u_{h}^{\prime}(x)\right)-\gamma F\left(x, s_{L}\right)-(1-\gamma) F\left(x, s_{U}\right)\right] d x\right| \\
& \quad \leq\left[\frac{\beta_{1}}{24}+\frac{\beta_{2}}{24\left(s_{U}-s_{L}\right)}+\frac{1}{2\left(s_{U}-s_{L}\right)}\left(1-\frac{h^{2}}{24}\right)^{1 / 2} \int_{0}^{1}\left|G^{\prime \prime}(x)\right| d x\right] h^{2} .
\end{aligned}
$$

Hence, for this example we obtain a higher-order error estimate for Theorem 1 when $F(x, s)$ is independent of $x$ and $M$ is even. When $M$ is odd and $F(x, s)$ is independent of $x$, we have that $u_{h}(0)=-u_{h}(1)$, and (5.13) and 
(5.14) are valid. So, when $M$ is odd and $F(x, s)$ is independent of $x$, Theorem 1 gives the optimal-order error estimate.

\section{BIBLIOGRAPHY}

1. R. A. Adams, Sobolev spaces, Academic Press, New York, 1975.

2. J. M. Ball, P. J. Holmes, R. D. James, R. L. Pego, and P. J. Swart, On the dynamics of fine structure, preprint, 1990.

3. J. M. Ball and R. D. James, Fine phase mixtures as minimizers of energy, Arch. Rational Mech. Anal. 100 (1987), 13-52.

4. _ Experimental tests of a theory of fine microstructure, preprint, 1990.

5. M. Chipot and D. Kinderlehrer, Equilibrium configurations of crystals, Arch. Rational Mech. Anal. 103 (1988), 237-277.

6. C. Collins, D. Kinderlehrer, and M. Luskin, Numerical approximation of the solution of a variational problem with a double well potential, SIAM J. Numer. Anal. 28 (1991), 321-332.

7. C. Collins and M. Luskin, The computation of the austenitic-martensitic phase transition, Partial Differential Equations and Continuum Models of Phase Transitions (M. Rascle, D. Serre, and M. Slemrod, eds.), Lecture Notes in Phys., vol. 344, Springer-Verlag, Berlin and New York, 1989, pp. 34-50.

8. __ Computational results for phase transitions in shape memory materials, Smart Materials, Structures, and Mathematical Issues (C. Rogers, ed.), Technomic Publishing Co., Lancaster, PA, 1989, pp. 198-215.

9. __ Numerical modeling of the microstructure of crystals with symmetry-related variants, Proceedings of the ARO US-Japan Workshop on Smart/Intelligent Materials and Systems, Technomic Publishing Co., Lancaster, PA, 1990, pp. 309-318.

10. R. E. Edwards, Functional analysis, Holt, Rinehart, and Winston, New York, 1965.

11. J. L. Ericksen, Some constrained elastic crystals, Material Instabilities in Continuum Mechanics and Related Problems (J. M. Ball, ed.), Oxford Univ. Press, Oxford, 1987, pp. 119137.

12. __ Constitutive theory for some constrained elastic crystals, Internat. J. Solids and Structures 22 (1986), 951-964.

13. I. Fonseca, Variational methods for elastic crystals, Arch. Rational Mech. Anal. 97 (1985), 189-220.

14. Math. Pures Appl. 67 (1988), 175-195.

15. D. A. French, On the convergence of finite element approximations of a relaxed variational problem, SIAM J. Numer. Anal. 27 (1990), 419-436.

16. R. James, Basic principles for the improvement of shape-memory and related materials, Smart Materials, Structures, and Mathematical Issues (C. Rogers, ed.), Technomic Publishing Co., Lancaster, PA, 1989.

17. R. D. James, Microstructure and weak convergence, Material Instabilities in Continuum Mechanics and Related Problems (J. M. Ball, ed.), Oxford Univ. Press, Oxford, 1987, pp. 175-196.

18. R. D. James and D. Kinderlehrer, Theory of diffusionless phase transitions, Partial Differential Equations and Continuum Models of Phase Transitions (M. Rascle, D. Serre, and M. Slemrod, eds.), Lecture Notes in Phys., vol. 344,, Springer-Verlag, Berlin, and New York, 1989, pp. 51-84.

19. D. Kinderlehrer, Remarks about equilibrium configurations of crystals, Material Instabilities in Continuum Mechanics and Related Problems (J. M. Ball, ed.), Oxford Univ. Press, Oxford, 1987, pp. 217-242. 
20. R. Kohn, The relationship between linear and nonlinear variational models of coherent phase transitions, Proceedings of the Seventh Army Conference on Applied Mathematics and Computing (F. Dressel, ed.), West Point, 1989.

21. W. Rudin, Real and complex analysis, 3rd ed., McGraw-Hill, New York, 1987.

22. L. Tartar, Compensated compactness and applications to partial differential equations, Nonlinear Analysis and Mechanics: Heriot-Watt Symposium IV, Research Notes 39 (R. J. Knops, ed.), Pitman, London, 1978, pp. 136-212.

Department of Mathematics, University of Michigan, Ann Arbor, Michigan 48109

School of Mathematics, University of Minnesota, Minneapolis, Minnesota 55455 